\title{
Statistical analysis of time-resolved emission from ensembles of semiconductor quantum dots: Interpretation of exponential decay models
}

Van Driel, A.F.; Nikolaev, I.S.; Vergeer, P.; LodahI, Peter; Vanmaelkelbergh, D.; Vos, W.L.

Published in:

Physical Review B Condensed Matter

Link to article, DOI:

10.1103/PhysRevB.75.035329

Publication date:

2007

Document Version

Publisher's PDF, also known as Version of record

Link back to DTU Orbit

Citation (APA):

Van Driel, A. F., Nikolaev, I. S., Vergeer, P., Lodahl, P., Vanmaelkelbergh, D., \& Vos, W. L. (2007). Statistical analysis of time-resolved emission from ensembles of semiconductor quantum dots: Interpretation of exponential decay models. Physical Review B Condensed Matter, 75(3), 035329.

https://doi.org/10.1103/PhysRevB.75.035329

\section{General rights}

Copyright and moral rights for the publications made accessible in the public portal are retained by the authors and/or other copyright owners and it is a condition of accessing publications that users recognise and abide by the legal requirements associated with these rights.

- Users may download and print one copy of any publication from the public portal for the purpose of private study or research.

- You may not further distribute the material or use it for any profit-making activity or commercial gain

- You may freely distribute the URL identifying the publication in the public portal 


\title{
Statistical analysis of time-resolved emission from ensembles of semiconductor quantum dots: Interpretation of exponential decay models
}

\author{
A. F. van Driel, ${ }^{1}$ I. S. Nikolaev, ${ }^{2,3}$ P. Vergeer, ${ }^{1}$ P. Lodahl, ${ }^{3,4}$ D. Vanmaekelbergh, ${ }^{1}$ and W. L. Vos $2,3, *$ \\ ${ }^{1}$ Debye Institute, Utrecht University, P.O. Box 80 000, 3508 TA Utrecht, The Netherlands \\ ${ }^{2}$ Center for Nanophotonics, FOM Institute for Atomic and Molecular Physics (AMOLF), 1098 SJ Amsterdam, The Netherlands \\ ${ }^{3}$ Complex Photonic Systems (COPS), Department of Science and Technology and MESA + Research Institute, \\ University of Twente, 7500 AE Enschede, The Netherlands \\ ${ }^{4}$ COM DTU Department of Communications, Optics, and Materials, Nano DTU, Technical University of Denmark, Lyngby, Denmark
}

(Received 22 June 2006; revised manuscript received 30 October 2006; published 22 January 2007)

\begin{abstract}
We present a statistical analysis of time-resolved spontaneous emission decay curves from ensembles of emitters, such as semiconductor quantum dots, with the aim of interpreting ubiquitous non-single-exponential decay. Contrary to what is widely assumed, the density of excited emitters and the intensity in an emission decay curve are not proportional, but the density is a time integral of the intensity. The integral relation is crucial to correctly interpret non-single-exponential decay. We derive the proper normalization for both a discrete and a continuous distribution of rates, where every decay component is multiplied by its radiative decay rate. A central result of our paper is the derivation of the emission decay curve when both radiative and nonradiative decays are independently distributed. In this case, the well-known emission quantum efficiency can no longer be expressed by a single number, but is also distributed. We derive a practical description of non-single-exponential emission decay curves in terms of a single distribution of decay rates; the resulting distribution is identified as the distribution of total decay rates weighted with the radiative rates. We apply our analysis to recent examples of colloidal quantum dot emission in suspensions and in photonic crystals, and we find that this important class of emitters is well described by a log-normal distribution of decay rates with a narrow and a broad distribution, respectively. Finally, we briefly discuss the Kohlrausch stretched-exponential model, and find that its normalization is ill defined for emitters with a realistic quantum efficiency of less than $100 \%$.
\end{abstract}

DOI: 10.1103/PhysRevB.75.035329

PACS number(s): 78.47.+p, 78.67.Hc, 78.55.Et

\section{INTRODUCTION}

Understanding the decay dynamics of excited states in emitters such as semiconductor quantum dots is of key importance for getting insight into many physical, chemical, and biological processes. For example, in biophysics the influence of Förster resonance energy transfer on the decay dynamics of donor molecules is studied to quantify molecular dynamics. ${ }^{1,2}$ In cavity quantum electrodynamics, modification of the density of states (DOS) is quantified by measuring the decay dynamics of light sources. According to Fermi's golden rule the radiative decay rate is proportional to the DOS at the location of the emitter. ${ }^{3}$ Nanocrystalline quantum dots, ${ }^{2,4,5}$ atoms, ${ }^{6,7}$ and dye molecules ${ }^{8,9}$ are used as light sources in a wide variety of systems. Examples of such systems are many different kinds of photonic materials, including metallic and dielectric mirrors, ${ }^{5-9}$ cavities, ${ }^{10}$ metallic films, ${ }^{11,12}$ and two-, ${ }^{13,14}$ and three-dimensional ${ }^{15}$ photonic crystals.

Figure 1 shows how observable parameters are related to the decay of an excited state $X^{*}$ to the ground state $X$. In photoluminescence lifetime measurements the decay of the number of excited emitters is probed by recording a photoluminescence decay curve $[f(t)]$. The number of excited emitters $c(t)$ can be probed directly by transient absorption measurements ${ }^{16-18}$ and nonradiative decay $(g-f)(t)$ can be recorded with photothermal techniques ${ }^{19,20}$ (see Fig. 1). $g(t)$ is here defined as the total intensity, i.e., the sum of the radiative and nonradiative processes. In this paper we discuss photoluminescence lifetime measurements, which are generally recorded by time-correlated single-photon counting. ${ }^{1}$ The decay curve $f(t)$ consists of a histogram of the distribution of arrival times of single photons after many excitationdetection cycles. ${ }^{1}$ The histogram is modelled with a decay function from which the decay time of the process is deduced.

In the simplest case when the system is characterized by a single decay rate $\Gamma$, the decay curve is described by a singleexponential function. However, in many cases the decay is much more complex and strongly differs from singleexponential decay. ${ }^{4,15,16,21-25}$ This usually means that the decay is characterized by a distribution of rates instead of a

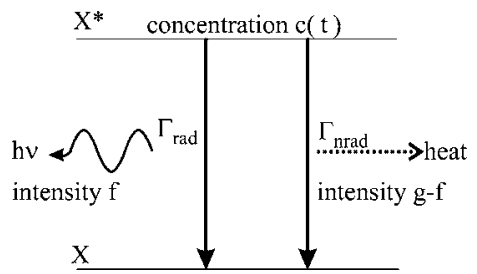

FIG. 1. Schematic of the relation between decay of an excited state $X^{*}$ to the ground state $X$ and experimental observable parameters. The density of emitters in the excited state is equal to $c(t)$ and can be probed by transient absorption. The emitted light intensity as a function of time $f(t)$ is recorded in luminescence decay measurements. In photothermal measurements the released heat $(g-f)(t)$ after photoexcitation is detected. $g(t)$ describes the total decay, i.e., the sum of the radiative and the nonradiative decay. 
single rate. ${ }^{46}$ For example, ensembles of quantum dots in photonic crystals experience the spatial and orientational variations of the projected local DOS (LDOS) explaining the non-single-exponential character of the decay. ${ }^{26}$ It is a general problem to describe such relaxation processes which do not follow a simple single-exponential decay. Sometimes double- and triple-exponential models are justified on the basis of prior knowledge of the emitters. ${ }^{1}$ However, in many cases no particular multiexponential model can be anticipated on the basis of physical knowledge of the system studied and a decision is made on the basis of the quality of fit.

Besides multiexponential models, the stretched-exponential model or Kohlrausch function ${ }^{27}$ is frequently applied. The stretched-exponential function has been applied to model diffusion processes ${ }^{28}$ dielectric relaxation, ${ }^{29}$ capacitor discharge, ${ }^{30}$ optical Kerr effect experiments, ${ }^{31}$ and luminescence decay. ${ }^{32-34}$ The physical origin of the apparent stretched-exponential decay in many processes remains a source of intense debate. ${ }^{35-37}$

Surprisingly, in spite of the rich variety of examples where non-single-exponential decay appears, there is no profound analysis of the models available in the literature. Therefore, we present in this paper a statistical analysis of time-resolved spontaneous emission decay curves from ensembles of emitters with the aim of interpreting ubiquitous non-single-exponential decay. Contrary to what is widely assumed, the density of excited emitters $c(t)$ and the intensity in an emission decay curve $[f(t)$ or $g(t)]$ are not proportional, but the density is a time integral of the intensity. The integral relation is crucial to correctly interpret non-singleexponential decay. We derive the proper normalization for both a discrete and a continuous distribution of rates, where every decay component is multiplied by its radiative decay rate. A central result of our paper is the derivation of the emission decay curve $f(t)$ when both radiative and nonradiative decays are independently distributed. In this most general case, the well-known emission quantum efficiency is also distributed. Distributed radiative decay is encountered in photonic media, ${ }^{26}$ while distributed nonradiative decay has been reported for colloidal quantum dots ${ }^{32,34}$ and powders doped with rare earths. ${ }^{38}$ We derive a practical description of non-single-exponential emission decay curves in terms of the distribution of total decay rates weighted with the radiative rates. Analyzing decay curves in terms of distributions of decay rates has the advantage that information on physically interpretable rates is readily available, as opposed to the previously reported analysis in terms of lifetimes. We apply our analysis to recent examples of colloidal quantum dot emission in suspensions and in photonic crystals. We find excellent agreement with a log-normal distribution of decay rates for such quantum dots. In the final section, we discuss the Kohlrausch stretched-exponential model, and find that its normalization is ill defined for emitters with a realistic quantum efficiency of less than $100 \%$.

\section{DECAY MODELS}

\section{A. Relation between the concentration of emitters and the decay curve}

A decay curve is the probability density of emission which is therefore modeled with a so-called probability den-

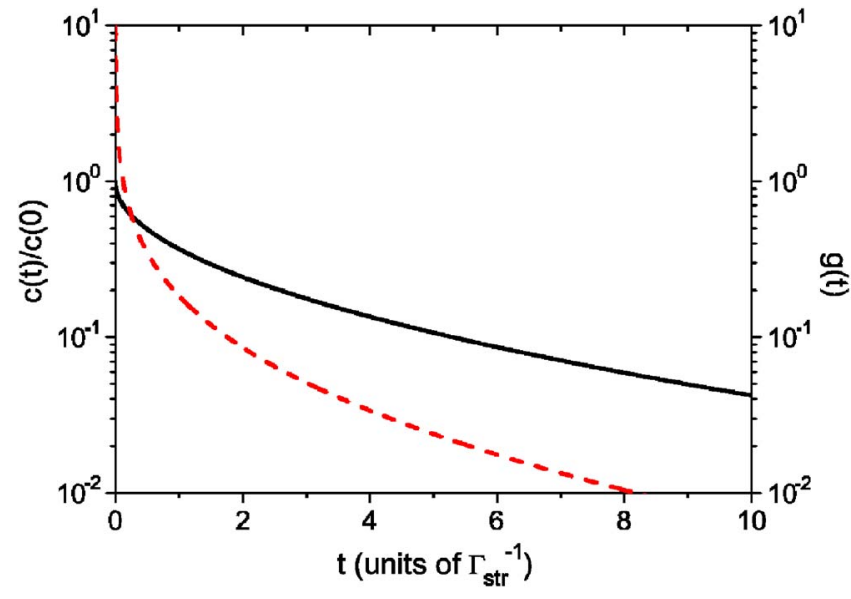

FIG. 2. (Color online) Plot of a non-single-exponential decay of the fraction $c(t) / c(0)$ (black solid curve, left axis) and the corresponding total intensity decay curve $g(t)$ (red dashed curve, right axis). The curves that describe the fraction of excited emitters and the corresponding intensity decay curve are strongly different. In this example, $c(t) / c(0)$ is the Kohlrausch stretched-exponential decay of the fraction [Eq. (19), black solid curve] and $g(t)$ the corresponding decay curve [Eq. (20), red dashed curve]. We have taken $\beta=0.5$ and $\Gamma_{\text {str }}=1$.

sity function. ${ }^{39}$ This function tends to zero in the limit $t$ $\rightarrow \infty$. The decay of the fraction of excited emitters $c\left(t^{\prime}\right) / c(0)$ at time $t^{\prime}$ is described with a reliability function or cumulative distribution function $\left[1-c\left(t^{\prime}\right) / c(0)\right] \cdot{ }^{39}$ Here $c(0)$ is the concentration of excited emitters at $t^{\prime}=0$. The reliability function tends to 1 in the limit $t^{\prime} \rightarrow \infty$ and to zero in the limit $t^{\prime} \rightarrow 0$. The fraction of excited emitters and the decay curve, i.e., the reliability function and the probability density function, ${ }^{39}$ are related as follows:

$$
\int_{0}^{t^{\prime}} g(t) d t=1-\frac{c\left(t^{\prime}\right)}{c(0)}
$$

Physically this equation means that the decrease of the concentration of excited emitters at time $t^{\prime}$ is equal to the integral of all previous decay events, or equivalently, the total intensity $g(t)$ is proportional to the time derivative of the fraction of excited emitters. As an illustration, Fig. 2 shows a non-single-exponential decay function simultaneously with the corresponding decay curve. It is clear that the two curves are strongly different. In many reports, however, the distinction between the reliability function and the probability density function is neglected: the intensity of the decay curve $g(t)$ is taken to be directly proportional to the fraction of excited emitters $c\left(t^{\prime}\right) / c(0)$. This proportionality holds only for single-exponential decay and not for non-singleexponential decay, which has important consequences for the interpretation of non-single-exponential decay.

\section{B. Single-exponential decay}

In this section, we will illustrate some concepts with the well-known single-exponential model. We will also indicate which features of single-exponential decay will break down 
in the general case of non-single-exponential decay. It is well known that in case of first-order kinetics the rate of decrease of the concentration is constant in time:

$$
\frac{d c\left(t^{\prime}\right)}{d t^{\prime}}=-\Gamma c\left(t^{\prime}\right)
$$

where $\Gamma$ is the decay rate of the process. As a consequence, the concentration $c\left(t^{\prime}\right)$ decreases single-exponentially in time:

$$
\frac{c\left(t^{\prime}\right)}{c(0)}=\exp \left(-\Gamma t^{\prime}\right)
$$

The mathematical expression for the luminescence decay curve can be obtained by inserting Eq. (3) into Eq. (1), where $\Gamma$ is identified with the total decay rate $\Gamma_{t o t}$, resulting in

$$
g(t)=\Gamma_{\text {rad }} \exp \left(-\Gamma_{\text {tot }} t\right)+\Gamma_{\text {nrad }} \exp \left(-\Gamma_{t o t} t\right)
$$

where $\Gamma_{\text {rad }}$ is the radiative decay rate, $\Gamma_{\text {nrad }}$ is the nonradiative decay rate, and $\Gamma_{t o t}$ is the total decay rate with $\Gamma_{t o t}$ $=\Gamma_{\text {rad }}+\Gamma_{\text {nrad }}$. In a luminescence decay measurement the recorded signal is proportional to the first term of $g(t)$ only, which is $f(t)$ :

$$
f(t)=\alpha \Gamma_{\text {rad }} \exp \left(-\Gamma_{t o t} t\right)
$$

and therefore a single-exponential luminescence decay process is modeled with Eq. (5). The preexponential factor $\alpha$ is usually taken as an adjustable parameter, and it is related to several experimental parameters, i.e., the number of excitation-emission cycles in the experiment, the photoncollection efficiency, and the concentration of the emitter. Henceforth $\alpha$ will be omitted in our analysis. A comparison between Eqs. (5) and (3) shows that in the case of pure single-exponential decay neglect of the distinction between the reliability function [Eq. (3)] and the probability density function [Eq. (5)] has no important consequences, since both the fraction and the decay curve are single exponentials. As Fig. 2 shows, this approximation breaks down in the case of non-single-exponential decay.

Figure 3 shows a luminescence decay curve of a dilute suspension of CdSe quantum dots in chloroform at a wavelength of $\lambda=650 \pm 5 \mathrm{~nm},{ }^{40}$ with the number of counts on the ordinate and the time on the abscissa. Clearly, the data agree well with single-exponential decay as indicated by the quality of fit $\chi_{r}^{2}$ of 1.12 , close to the ideal value of 1 . This means that all individual quantum dots that emit light in this particular wavelength range do so with the same rate of $1 / 39.0 \mathrm{~ns}^{-1}$. It appears that the rate of emission strongly depends on the emission frequency and that it is determined by the properties of the bulk semiconductor crystal. ${ }^{40}$

Since $f(t)$ as given by Eq. (5) is a probability density function, the probability of emission in a certain time interval can be deduced by integration. The total probability for emission at all times between $t=0$ and $t \rightarrow \infty$ is given by

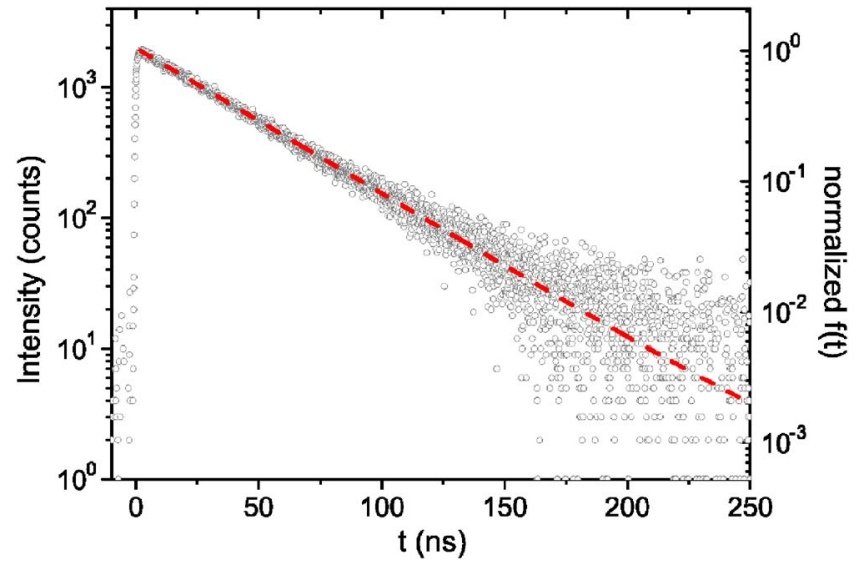

FIG. 3. (Color online) Luminescence decay curve of emission from a dilute suspension of CdSe quantum dots (open dots, left axis). Data were collected at the red side of the emission maximum of the suspension, at $\lambda=650 \pm 5 \mathrm{~nm}$. Single-exponential modeling (red dashed curve, right axis) yields a decay time of $39.0 \pm 2.8 \mathrm{~ns}$ and a $\chi_{r}^{2}$ of 1.12 . The average photon arrival time $\langle t\rangle$, calculated with Eq. (7), is $39.1 \mathrm{~ns}$.

$$
\int_{0}^{\infty} f(t) d t=\int_{0}^{\infty} \Gamma_{r a d} \exp \left(-\Gamma_{t o t} t\right) d t=\frac{\Gamma_{r a d}}{\Gamma_{t o t}}
$$

which is equal to the luminescence quantum efficiency. The luminescence quantum efficiency is defined as the probability of emission after excitation. ${ }^{1}$ The correct recovery of this result in Eq. (6) shows that Eq. (5) is properly normalized.

The average arrival time of the emitted photons or the average decay time can be calculated by taking the first moment of Eq. (5):

$$
\langle t\rangle=\tau_{a v}=\frac{\int_{0}^{\infty} f(t) t d t}{\int_{0}^{\infty} f(t) d t}=\frac{1}{\Gamma_{t o t}} .
$$

Only in the case of single-exponential decay is the average decay time $\langle t\rangle$ equal to the inverse of the total decay rate $\Gamma_{t o t}$. The average arrival time for the data in Fig. 3 was $\langle t\rangle$ $=39.1 \mathrm{~ns}$, very close to the value of $39.0 \pm 2.8 \mathrm{~ns}$ obtained from single-exponential modeling, which further confirms the single-exponential character of the decay of quantum dots in suspension.

\section{Discrete distribution of decay rates}

In contrast to the example shown in Fig. 3, there are many cases in which decay curves cannot be modeled with a single-exponential function. As an example, Fig. 4 shows a strongly non-single-exponential decay curve of spontaneous emission from CdSe quantum dots in an inverse opal photonic crystal. ${ }^{15,26}$ If a non-single-exponential decay curve is modeled with a sum of single exponentials, the decay curve has the following form: 


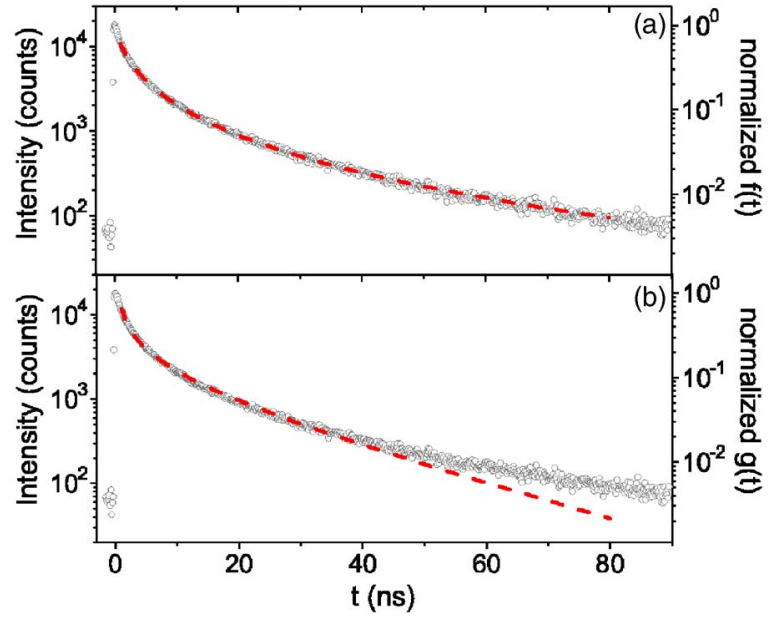

FIG. 4. (Color online) Luminescence decay curve of emission from CdSe quantum dots in a titania inverse opal photonic crystal (dots, left axis). The lattice parameter of the titania inverse opal was $340 \mathrm{~nm}$ and the emission wavelength $\lambda=595 \mathrm{~nm}$. (a) A log-normal distribution of rates [Eqs. (17) and (18), red dashed curve, right axis] models the data extremely well $\left(\chi_{r}^{2}=1.17\right)$. The $\Gamma_{m f}$ is $91.7 \mu \mathrm{s}^{-1}\left(1 / \Gamma_{m f}=10.9 \mathrm{~ns}\right)$ and the width of the distribution $\Delta \Gamma$ is $0.57 \mathrm{~ns}^{-1}$. (b) In contrast, a Kohlrausch stretched-exponential model (red dashed curve, right axis) does not fit the data $\left(\chi_{r}^{2}=60.7\right)$. The stretched-exponential curve corresponds to $\Gamma_{s t r}=96.2 \mu \mathrm{s}^{-1}\left(1 / \Gamma_{s t r}\right.$ $=10.4 \mathrm{~ns}$ ), an average decay time $\langle t\rangle$ of $31.1 \mathrm{~ns}$, and a $\beta$ value of 0.42 .

$$
f(t)=\frac{1}{c(0)} \sum_{i=1}^{n} c_{i} \Gamma_{r a d, i} \exp \left(-\Gamma_{t o t, i} t\right)
$$

where $n$ is the number of different emitters (or alternatively the number of different environments of single emitters $\left.{ }^{26}\right), c_{i}$ is the concentration of emitters that have a radiative decay rate $\Gamma_{r a d, i}$, and $c(0)$ is the concentration of excited emitters at $t=0$, i.e., the sum of all concentrations $c_{i}$. When the different fractions (or environments) are distributed in a particular way, a distribution function $\rho\left(\Gamma_{t o t}\right)$ may be used. Such a function describes the distribution or concentration of the emitters over the emission decay rates at time $t=0$. The fraction of emitters with a total decay rate $\Gamma_{t o t, i}$ is given by

$$
\begin{aligned}
\frac{c_{i}}{c(0)} & =\frac{1}{c(0)} \frac{\left[c\left(\Gamma_{t o t, i-1}\right)+c\left(\Gamma_{t o t, i+1}\right)\right]}{2} \\
& =\frac{1}{2} \int_{\Gamma_{t o t, i-1}}^{\Gamma_{t o t, i+1}} \rho\left(\Gamma_{t o t}\right) d \Gamma_{t o t} \\
& =\rho\left(\Gamma_{t o t, i}\right) \Delta \Gamma_{t o t},
\end{aligned}
$$

where $\rho\left(\Gamma_{t o t, i}\right)$ expresses the distribution of the various components $i$ over the rates $\Gamma_{t o t, i}$ and has units of inverse rate $s$. $\Delta \Gamma_{t o t}$ is the separation between the various components $i$ in the sum. The decay curve now has the following mathematical form:

$$
f(t)=\sum_{i=1}^{n} \Delta \Gamma_{t o t} \rho\left(\Gamma_{t o t, i}\right) \Gamma_{r a d, i} \exp \left(-\Gamma_{t o t, i} t\right) .
$$

It is important to note that in Eq. (10) every component in the sum is correctly normalized since every component is multiplied by its radiative decay rate $\Gamma_{r a d, i}$.

\section{Continuous distribution of decay rates}

For infinitesimal values of $\Delta \Gamma_{\text {tot }}$, Eq. (10) can be written as an integral:

$$
f(t)=\int_{0}^{\infty} \Gamma_{r a d}\left(\Gamma_{t o t}\right) \rho\left(\Gamma_{t o t}\right) \exp \left(-\Gamma_{t o t} t\right) d \Gamma_{t o t} .
$$

In the case of single-exponential decay the distribution function is strongly peaked around a central $\Gamma_{\text {tot }}$ value, i.e., the distribution function is a Dirac $\delta$ function. Inserting a Dirac $\delta$ function into Eq. (11) recovers Eq. (5):

$$
\begin{aligned}
f(t) & =\int_{0}^{\infty} \Gamma_{r a d^{\prime}} \delta\left(\Gamma_{t o t}-\Gamma_{t o t^{\prime}}\right) \exp \left(-\Gamma_{t o t} t\right) d \Gamma_{t o t} \\
& =\Gamma_{r a d^{\prime}} \exp \left(-\Gamma_{t o t^{\prime}} t\right) .
\end{aligned}
$$

This result confirms that the generalization to Eq. (11) is correct since it yields the correctly normalized singleexponential functions.

In Eq. (11) it is tacitly assumed that for every $\Gamma_{t o t}$ there is one $\Gamma_{\text {rad }}$ : the function $\Gamma_{\text {rad }}\left(\Gamma_{t o t}\right)$ relates each $\Gamma_{t o t}$ to exactly one $\Gamma_{\text {rad }}$. In general both $\Gamma_{t o t}$ and $\Gamma_{\text {rad }}$ vary independently, and Eq. (11) is generalized to

$f(t)=\int_{0}^{\infty}\left(\int_{0}^{\Gamma_{t o t}} d \Gamma_{r a d} \rho_{\Gamma_{t o t}}\left(\Gamma_{r a d}\right) \Gamma_{r a d}\right) \rho\left(\Gamma_{t o t}\right) \exp \left(-\Gamma_{t o t} t\right) d \Gamma_{t o t}$,

where $\rho_{\Gamma_{t o t}}\left(\Gamma_{r a d}\right)$ is the normalized distribution of $\Gamma_{r a d}$ at constant $\Gamma_{\text {tot }}$. For every $\Gamma_{\text {tot }}$ the integration is performed over all radiative rates; a distribution of $\Gamma_{\text {rad }}$ is taken into account for every $\Gamma_{t o t}$. Equation (13) is the most general expression of a luminescence decay curve and a central result of our paper. From this equation every decay curve with a particular distribution of rates can be recovered. An example described by Eq. (13) is an ensemble of quantum dots in a photonic crystal. In photonic crystals the local density of optical states varies with the location in the crystal and the distribution of dipole orientations of the emitters. ${ }^{41}$ Therefore, an ensemble of emitters with a certain frequency emits light with a distribution of radiative rates $\Gamma_{\text {rad }}$. In addition, when an ensemble of emitters has a distributed $\Gamma_{t o t}$ and a single radiative rate $\Gamma_{r a d}$, i.e., $\rho_{\Gamma_{\text {tot }}}\left(\Gamma_{r a d}\right)$ is a $\delta$ function, then Eq. (13) reduces to Eq. (11). Even though the nonradiative rates may still be distributed, Eq. (11) suffices to describe the decay curve since for every $\Gamma_{\text {tot }}$ there is only one $\Gamma_{\text {rad }}$. Such a situation appears, for example, with powders doped with rare earth ions $^{38}$ and with polymer films doped with quantum dots. ${ }^{32,34}$

Interestingly, an ensemble of emitters with a distribution of rates $\Gamma_{t o t}$ is not completely characterized by a single value of the quantum efficiency [as opposed to Eq. (6) for single- 
exponential decay]. In such an ensemble, the quantum efficiency is distributed, since each $\Gamma_{t o t}$ is associated with a distribution of radiative rates $\Gamma_{\text {rad }}$. The average quantum efficiency $\langle\eta\rangle$ can be calculated by integrating Eq. (13) for all times:

$$
\begin{aligned}
\langle\eta\rangle= & \int_{0}^{\infty} f(t) d t \\
= & \int_{0}^{\infty} \int_{0}^{\infty}\left(\int_{0}^{\Gamma_{t o t}} d \Gamma_{r a d} \rho_{\Gamma_{t o t}}\left(\Gamma_{r a d}\right) \Gamma_{r a d}\right) \\
& \times \rho\left(\Gamma_{t o t}\right) \exp \left(-\Gamma_{t o t} t\right) d \Gamma_{t o t} d t .
\end{aligned}
$$

Most often, detailed information on the relation between $\Gamma_{t o t}$ and $\Gamma_{\text {rad }}$ is not available. Then, direct modeling with a distribution of decay rates is applied. ${ }^{21,23,24,37,42}$ This approach has a major advantage over modeling with a stretched-exponential function, where it is complicated to deduce the distribution of decay rates (see below). A function of the following form is used to model the non-singleexponential decay curve:

$$
f(t)=\int_{0}^{\infty} \sigma\left(\Gamma_{t o t}\right) \exp \left(-\Gamma_{t o t} t\right) d \Gamma_{t o t}
$$

In Eq. (15) the various components are not separately normalized as in Eq. (13). Modeling with Eq. (15) boils down to using an infinite series of single exponentials which are expressed with only a few free parameters. The form of the distribution can usually not be predicted and a decision is made on the basis of the quality of fit. While a good fit does not prove that the chosen distribution is unique, it does extract direct physical information from the non-singleexponential decay on an ensemble of emitters and their environment. ${ }^{26}$

It is widely assumed that $\sigma(\Gamma)$ is equal to the distribution of total rates. ${ }^{21,23,24,43,44}$ A comparison with Eq. (13) shows that this is not true and reveals that $\sigma(\Gamma)$ contains information about both the radiative and nonradiative rates:

$$
\sigma\left(\Gamma_{t o t}\right)=\rho\left(\Gamma_{t o t}\right) \int_{0}^{\Gamma_{t o t}} \rho_{\Gamma_{t o t}}\left(\Gamma_{r a d}\right) \Gamma_{r a d} d \Gamma_{r a d}
$$

Thus $\sigma(\Gamma)$ is the distribution of total decay rates weighted by the radiative rates. This conclusion demonstrates the practical use of Eq. (13): the equation allows us to completely interpret the distribution of rates found by modeling with Eq. (15). Such a complete interpretation has not been reported before to our knowledge.

\section{E. Log-normal distribution of decay rates}

Distribution functions that can be used for $\sigma(\Gamma)$ are (sums of) normal, Lorentzian, and log-normal distribution functions. In Fig. 4(a) the luminescence decay curve of quantum dots is successfully modeled with Eq. (15), with a lognormal distribution of the rate $\Gamma$,

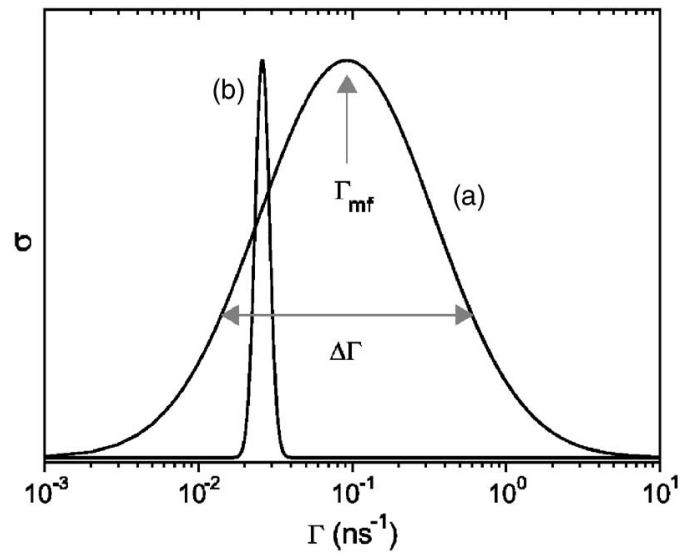

FIG. 5. Log-normal distribution of $\Gamma$. This distribution was modeled to the data of Fig. 4 (curve $a$, quantum dots in photonic crystal) and Fig. 3 (curve $b$, quantum dots in a diluted suspension), with $\Gamma_{m f}$ and $\Delta \Gamma$ as adjustable parameters. For curve $a \Gamma_{m f}$ is $91.7 \mu \mathrm{s}^{-1}\left(1 / \Gamma_{m f}=10.9 \mathrm{~ns}\right)$ and the width of the distribution $\Delta \Gamma$ is $0.57 \mathrm{~ns}^{-1}$ and for curve $b \Gamma_{m f}$ is $25.8 \mu \mathrm{s}^{-1}\left(1 / \Gamma_{m f}=38.8 \mathrm{~ns}\right)$ and the width of the distribution $\Delta \Gamma$ is $0.079 \mathrm{~ns}^{-1}$.

$$
\sigma(\Gamma)=A \exp \left[-\left(\frac{\ln \Gamma-\ln \Gamma_{m f}}{\gamma}\right)^{2}\right],
$$

where $A$ is the normalization constant and $\Gamma_{m f}$ is the most frequent rate constant (see Fig. 5). $\gamma$ is related to the width of the distribution:

$$
\Delta \Gamma=2 \Gamma_{m f} \sinh (\gamma),
$$

where $\Delta \Gamma$ is equal to the width of the distribution at $1 / e$. The most frequent rate constant $\Gamma_{m f}$ and $\gamma$ are adjustable parameters, only one extra adjustable parameter compared to a single-exponential model. Clearly, this model [Eqs. (15) and (17)] describes our non-single-exponential experimental data extremely well. $\chi_{r}^{2}$ was $1.17, \Gamma_{m f}$ was $91.7 \mu \mathrm{s}^{-1}\left(1 / \Gamma_{m f}\right.$ $=10.9 \mathrm{~ns}$ ), and the width of the distribution $\Delta \Gamma$ was $0.57 \mathrm{~ns}^{-1}$. In addition to $\Gamma_{m f}$ and $\Delta \Gamma$, an average decay rate can be deduced from the log-normal distribution in Fig. 5. However, this average is biased since the various components are weighted with their quantum efficiency, as shown in Eq. (16).

Modeling with a log-normal distribution of decay rates yields direct and clear physical parameters, for instance the shape and width of the decay rate distribution. The lognormal function is plotted in Fig. 5 (curve $a$ ). The broad distribution of rates demonstrates the strongly non-singleexponential character of the decay curve. In Ref. 26 we were able to relate the width of this broad distribution to the spatial and orientational variations of the LDOS in inverse-opal photonic crystals.

The log-normal model was also modeled to the decay curve from quantum dots in suspension (Fig. 3). The distribution is plotted in Fig. 5 (curve $b$ ). $\Gamma_{m f}$ was $25.8 \mu \mathrm{s}^{-1}$ $\left(1 / \Gamma_{m f}=38.8 \mathrm{~ns}\right)$, close to the lifetime deduced from the single-exponential modeling of $39.0 \pm 2.8 \mathrm{~ns}$. The narrow width of the distribution $\Delta \Gamma$ of $0.079 \mathrm{~ns}^{-1}$ is in agreement with the single-exponential character of the decay curve. 


\section{F. Stretched-exponential decay}

In addition to the multiexponential models discussed in Secs. II C-II E, the Kohlrausch stretched-exponential decay model $^{27,29}$ is widely applied to model non-single-exponential decay curves. The fraction of excited emitters, i.e., the reliability function, of the Kohlrausch stretched-exponential model is equal to

$$
\frac{c\left(t^{\prime}\right)}{c(0)}=\exp \left[-\left(\Gamma_{s t r} t^{\prime}\right)^{\beta}\right],
$$

where $\beta$ is the stretch parameter, which varies between 0 and 1 , and $\Gamma_{\text {str }}$ the total decay rate in the case of stretchedexponential decay. The stretch parameter $\beta$ qualitatively expresses the underlying distribution of rates: a small $\beta$ means that the distribution of rates is broad and $\beta$ close to 1 implies a narrow distribution. The recovery of the distribution of rates in the case of stretched-exponential decay is mathematically complicated ${ }^{47}$ and feasible only for specific $\beta$ 's. ${ }^{22,29,35,36}$

The decay curve corresponding to a Kohlrausch stretchedexponential decay of the fraction $c\left(t^{\prime}\right) / c(0)$ can be deduced using Eqs. (19) and (1), and results in

$$
g(t)=\frac{\beta}{t}\left(\Gamma_{s t r} t\right)^{\beta} \exp \left[-\left(\Gamma_{s t r} t\right)^{\beta}\right]
$$

The normalization of Eq. (20) can, in analogy with Eq. (6), be deduced by integration for all times between $t=0$ and $t$ $\rightarrow \infty$, which yields 1 . Therefore, an important consequence is that Eq. (20) is correctly normalized only for emitters with a quantum yield of $1\left[\Gamma_{\mathrm{rad}}=\Gamma_{\text {tot }}\right.$ and $\left.f(t)=g(t)\right]$. It is not clear how normalization should be done in realistic cases with quantum yield less than $100 \%$. To the best of our knowledge, this problem has been overlooked in the literature.

The main advantage of modeling with a Kohlrausch stretched-exponential function is that the average decay time $\langle t\rangle$ can readily be calculated. The average decay time is equal to $^{29}$

$$
\langle t\rangle=\tau_{a v}=\frac{\int_{0}^{\infty} g(t) t d t}{\int_{0}^{\infty} g(t) d t}=\frac{1}{\Gamma_{s t r} \beta} \bar{\Gamma}\left(\frac{1}{\beta}\right),
$$

where $\bar{\Gamma}$ is the mathematical Gamma function. For the single-exponential limit of $\beta \rightarrow 1 \mathrm{Eq}$. (21) reduces to Eq. (7). Note again that in this average the various contributions are weighted with their quantum efficiency and that the average decay time $\langle t\rangle$ differs from $1 / \Gamma_{s t r}$ (see Sec. II E). Indeed, for the data in Fig. 4(b), Eq. (21) yielded an average decay time of $31.1 \mathrm{~ns}$, strongly different from the $1 / \Gamma_{s t r}$ value of $10.4 \mathrm{~ns}$, in contrast to the result [Eq. (5)] for singleexponential decay.

It is important to note that Eq. (21) is the average decay time $\langle t\rangle$ corresponding to the decay curve given by Eq. (20). In, for instance, Refs. 32 and 45 the average time given by
Eq. (21) has erroneously been associated with fluorescence decay described by Eq. (19).

In contrast to the single-exponential model, the reliability function and the probability density function of a stretched exponential do not have the same form (see Fig. 2); the probability density function contains a time-dependent prefactor. Therefore, the relation between the reliability function and the probability density function [Eq. (1)] has important consequences. For a $\beta$ value of 0.5 the average decay times of the reliability function [Eq. (19)] and of the probability density function [Eq. (20)] differ by more than a factor of 10 . Thus it is important to take into consideration whether Eq. (19) or Eq. (20) is used to describe the experimental photoluminescence decay curve. This is important, since in many reports, ${ }^{32-34,37,43,44}$ the luminescence decay curve is modeled with the time dependence of Eq. (19). We remark that, while Eq. (19) can be used to account for the deviation from single-exponential decay, it does not represent the true Kohlrausch function, but is simply an alternative model. We argue that using the Kohlrausch stretched exponential as a reliability function to model the fraction ${ }^{48} c\left(t^{\prime}\right) / c(0)$ implies that the proper probability density function, i.e., Eq. (20), must be used to model a luminescence decay curve. Figure 4(b) shows the modeling of experimental data with Eq. (20), with $\Gamma_{\text {str }}$ and $\beta$ as adjustable parameters. The $\beta$ value was 0.42 and $\Gamma_{s t r}$ was $96.2 \mu \mathrm{s}^{-1}\left(1 / \Gamma_{s t r}=10.4 \mathrm{~ns}\right)$. Modeling with a stretched exponential is obviously more satisfactory than a single exponential, but here fails at long times, reflected by the high $\chi_{r}^{2}$ value of 60.7 .

\section{CONCLUSIONS}

We have presented a statistical analysis of time-resolved spontaneous emission decay curves from ensembles of emitters, in particular colloidal quantum dots, with the aim of interpreting the ubiquitous non-single-exponential decay. Contrary to what is widely assumed, the density of excited emitters $c(t)$ and the intensity in an emission decay curve $[f(t)$ or $g(t)]$ are not proportional, but the density is a time integral of the intensity. The integral relation is crucial to correctly interpret non-single-exponential decay. We have derived the proper normalization for both a discrete and a continuous distribution of rates, where every decay component is multiplied by its radiative decay rate. A central result of our paper is the derivation of the emission decay curve $f(t)$ when both radiative and nonradiative decays are independently distributed [Eq. (13)]. In this case, the well-known emission quantum efficiency can no longer be expressed by a single number, but is also distributed. We derive a practical description of non-single-exponential emission decay curves in terms of a distribution of total decay rates weighted with the radiative rates. Analyzing decay curves in terms of decay rate distributions is opposite to the usual and widely reported analysis in terms of distributed lifetimes. We apply our analysis to recent examples of colloidal quantum dot emission in suspensions and in photonic crystals, and we find that 
this important class of emitters is well described by a lognormal distribution of decay rates with a narrow and a broad distribution, respectively. Finally, we briefly discuss the Kohlrausch stretched-exponential model; we deduce the average decay time and we find that its normalization is ill defined for emitters with a realistic quantum efficiency of less than $100 \%$.

\section{ACKNOWLEDGMENTS}

This work is part of the research program of both the "Stichting voor Fundamenteel Onderzoek der Materie (FOM)," and "Chemische Wetenschappen," which are financially supported by the "Nederlandse Organisatie voor Wetenschappelijk Onderzoek (NWO)."
*Electronic address: w.l.vos@utwente.nl; URL: http:// www.photonicbandgaps.com

${ }^{1}$ J. R. Lakowicz, Principles of Fluorescence Spectroscopy, 2nd ed. (Kluwer Academic/Plenum Publishers, New York, 1999).

${ }^{2}$ I. L. Medintz, H. T. Uyeda, E. R. Goldman, and H. Mattoussi, Nat. Mater. 4, 435 (2005).

${ }^{3}$ R. Loudon, The Quantum Theory of Light, 3rd ed., Oxford Science Publications (Oxford University Press, Oxford, 2001).

${ }^{4}$ S. A. Crooker, J. A. Hollingsworth, S. Tretiak, and V. I. Klimov, Phys. Rev. Lett. 89, 186802 (2002).

${ }^{5}$ J. Y. Zhang, X. Y. Wang, and M. Xiao, Opt. Lett. 27, 1253 (2002).

${ }^{6}$ K. H. Drexhage, J. Lumin. 1,2, 693 (1970).

${ }^{7}$ R. M. Amos and W. L. Barnes, Phys. Rev. B 55, 7249 (1997).

${ }^{8}$ N. Danz, J. Heber, A. Bräuer, and R. Kowarschik, Phys. Rev. A 66, 063809 (2002).

${ }^{9}$ S. Astilean and W. L. Barnes, Appl. Phys. B: Lasers Opt. 75, 591 (2002).

${ }^{10}$ M. Bayer, T. L. Reinecke, F. Weidner, A. Larionov, A. McDonald, and A. Forchel, Phys. Rev. Lett. 86, 3168 (2001).

${ }^{11}$ J. S. Biteen, D. Pacifici, N. S. Lewis, and H. A. Atwater, Nano Lett. 5, 1768 (2005).

${ }^{12}$ J. H. Song, T. Atay, S. F. Shi, H. Urabe, and A. V. Nurmikko, Nano Lett. 5, 1557 (2005).

${ }^{13}$ M. Fujita, S. Takahashi, Y. Tanaka, T. Asano, and S. Noda, Science 308, 1296 (2005).

${ }^{14}$ A. Kress, F. Hofbauer, N. Reinelt, M. Kaniber, H. J. Krenner, R. Meyer, G. Böhm, and J. J. Finley, Phys. Rev. B 71, 241304(R) (2005).

${ }^{15}$ P. Lodahl, A. F. van Driel, I. S. Nikolaev, A. Irman, K. Overgaag, D. Vanmaekelbergh, and W. L. Vos, Nature (London) 430, 654 (2004).

${ }^{16}$ P. Foggi, L. Pettini, I. Santa, R. Righini, and S. Califano, J. Phys. Chem. 99, 7439 (1995).

${ }^{17}$ V. I. Klimov and D. W. McBranch, Phys. Rev. Lett. 80, 4028 (1998).

${ }^{18}$ F. V. R. Neuwahl, P. Foggi, and R. G. Brown, Chem. Phys. Lett. 319, 157 (2000).

${ }^{19}$ A. Rosencwaig, Photoacoustics and Photoacoustic Spectroscopy (John Wiley \& Sons, New York, 1980).

${ }^{20}$ M. Grinberg, A. Sikorska, and A. Śliwiński, Phys. Rev. B 67, 045114 (2003).

${ }^{21}$ D. R. James and W. R. Ware, Chem. Phys. Lett. 126, 7 (1986).

${ }^{22}$ A. Siemiarczuk, B. D. Wagner, and W. R. Ware, J. Phys. Chem. 94, 1661 (1990)

${ }^{23}$ J. C. Brochon, A. K. Livesey, J. Pouget, and B. Valeur, Chem. Phys. Lett. 174, 517 (1990)

${ }^{24}$ J. Wlodarczyk and B. Kierdaszuk, Biophys. J. 85, 589 (2003).
${ }^{25}$ S. F. Wuister, A. van Houselt, C. D. M. Donega, D. Vanmaekelbergh, and A. Meijerink, Angew. Chem., Int. Ed. 43, 3029 (2004).

${ }^{26}$ I. S. Nikolaev, P. Lodahl, A. F. van Driel, A. F. Koenderink, and W. L. Vos, physics/0511133 (to be published).

${ }^{27}$ R. Kohlrausch, Ann. Phys. 91, 179 (1854).

${ }^{28}$ L. A. Deschenes and D. A. Vanden Bout, Science 292, 255 (2001).

${ }^{29}$ C. P. Lindsey and G. D. Patterson, J. Chem. Phys. 73, 3348 (1980).

${ }^{30}$ R. Kohlrausch, Ann. Phys. 91, 56 (1854).

${ }^{31}$ R. Torre, P. Bartolini, and R. Righini, Nature (London) 428, 296 (2004).

${ }^{32}$ G. Schlegel, J. Bohnenberger, I. Potapova, and A. Mews, Phys. Rev. Lett. 88, 137401 (2002).

${ }^{33}$ R. Chen, J. Lumin. 102, 510 (2003).

${ }^{34}$ B. R. Fisher, H. J. Eisler, N. E. Stott, and M. G. Bawendi, J. Phys. Chem. B 108, 143 (2004).

${ }^{35}$ D. L. Huber, Phys. Rev. B 31, 6070 (1985).

${ }^{36}$ F. Alvarez, A. Alegria, and J. Colmenero, Phys. Rev. B 44, 7306 (1991).

${ }^{37}$ M. Lee, J. Kim, J. Tang, and R. M. Hochstrasser, Chem. Phys. Lett. 359, 412 (2002).

${ }^{38}$ P. Vergeer, T. J. H. Vlugt, M. H. F. Kox, M. I. den Hertog, J. P. J. M. van der Eerden, and A. Meijerink, Phys. Rev. B 71, 014119 (2005).

${ }^{39}$ E. R. Dougherty, Probability and Statistics for the Engineering, Computing and Physical Sciences (Prentice-Hall, Englewood, NJ, 1990).

${ }^{40}$ A. F. van Driel, G. Allan, C. Delerue, P. Lodahl, W. L. Vos, and D. Vanmaekelbergh, Phys. Rev. Lett. 95, 236804 (2005).

${ }^{41}$ R. Sprik, B. A. van Tiggelen, and A. Lagendijk, Europhys. Lett. 35, 265 (1996).

${ }^{42}$ D. R. James, Y.-S. Liu, P. De Mayo, and W. R. Ware, Chem. Phys. Lett. 120, 460 (1985).

${ }^{43}$ K. C. Benny Lee, J. Siegel, S. E. D. Webb, S. Leveque-Fort, M. J. Cole, R. Jones, K. Dowling, M. J. Lever, and P. M. W. French, Biophys. J. 81, 1265 (2001).

${ }^{44}$ M. N. Berberan-Santos, E. N. Bodunov, and B. Valeur, Chem. Phys. 315, 171 (2005).

${ }^{45}$ J. Kalkman, H. Gersen, L. Kuipers, and A. Polman, Phys. Rev. B 73, 075317 (2006).

${ }^{46}$ In the case of strong coupling in cavity quantum electrodynamics, the decay of even a single emitter is not single exponential. Experimental situations where this may be encountered are emitters in a high-finesse cavity, or van Hove singularities in the LDOS of a photonic crystal.

${ }^{47}$ In case of the stretched-exponential model the distribution of the rates is unknown and is generally deduced by solving 
the following equation (Refs. 21, 32, 42, 43, and 44): $(\beta / t)\left(\Gamma_{s t r} t\right)^{\beta} \exp \left[-\left(\Gamma_{s t r} t\right)^{\beta}\right]=\int_{0}^{\infty} \sigma(\Gamma) \exp (-\Gamma t) d \Gamma$, where $\sigma(\Gamma)$ is the distribution function of the total decay rate weighted by $\Gamma_{\text {rad }}$. To deduce $\sigma(\Gamma)$ an inverse Laplace transform is applied. For $\beta \neq 0.5$ and $\beta \neq 1$ there is no analytical solution of this equation and therefore it is difficult to deduce the distribution function.
This difficulty can be circumvented by modeling directly with a known distribution function, as is shown in this paper.

${ }^{48}$ We remark that in Refs. 27 and 29 capacitor discharge and dielectric relaxation are studied, which are, in contrast to a fluorescence decay curve, indeed described by a reliability function. 